\title{
Role of higher-order Hermite polynomials in the central-moments-based lattice Boltzmann framework
}

\author{
Alessandro De Rosis* \\ Electric Ant Lab B.V., Science Park 400, NL-1098 XH Amsterdam, The Netherlands \\ Kai H. $\mathrm{Luo}^{\dagger}$ \\ Department of Mechanical Engineering, University College London, London WC1E 7JE, United Kingdom
}

(Received 23 August 2018; revised manuscript received 2 November 2018; published 2 January 2019)

\begin{abstract}
The cascaded lattice Boltzmann method decomposes the collision stage on a basis of central moments on which the equilibrium state is assumed equal to that of the continuous Maxwellian distribution. Such a relaxation process is usually considered as an assumption, which is then justified a posteriori by showing the enhanced Galilean invariance of the resultant algorithm. An alternative method is to relax central moments to the equilibrium state of the discrete second-order truncated distribution. In this paper, we demonstrate that relaxation to the continuous Maxwellian distribution is equivalent to the discrete counterpart if higher-order (up to sixth) Hermite polynomials are used to construct the equilibrium when the D3Q27 lattice velocity space is considered. Therefore, a theoretical a priori justification of the choice of the continuous distribution is formally provided for the first time.
\end{abstract}

DOI: 10.1103/PhysRevE.99.013301

\section{INTRODUCTION}

The lattice Boltzmann method (LBM) is a popular scheme to perform fluid flow simulations [1-6]. In short, fluid dynamics arises from the motion of distributions (or populations) of fictitious particles that collide and stream along the links of a fixed Cartesian lattice. The absence of necessity for moving meshes, the low intrinsic numerical dissipation and the efficiency of the algorithm represent the key pivotal features that promoted the success of the LBM.

The most common collision operator is the BhatnagarGross-Krook (BGK) procedure that forces all the populations to relax to an equilibrium state with a common unique rate [7]. Despite its popularity, this model manifests instability when large velocity gradients arise in the flow due to the presence of nonhydrodynamic ghost modes [8]. By decomposing the collision operator on a basis of raw moments, the multiple-relaxation-time (MRT) model has proved to enhance the stability of the LBM by properly damping high-order nonhydrodynamic modes [9] and acoustic waves [10]. However, the MRT trims the Galilean invariance, too, due to the representation of the collision in a frame at rest [11].

In 2006, Geier et al. [12] pointed out this issue. Specifically, they proposed to circumvent the problem by shifting the lattice directions by the local fluid velocity. By introducing the concept of central moments (CMs), they derived a collision operator that shows a pyramidal hierarchical structure, where the post-collision state of a certain moment at a given order depends on lower-order ones, hence the name "cascaded" kernel. An important assumption on the cascaded LBM is to

\footnotetext{
${ }^{*}$ Corresponding author: derosis.alessandro@icloud.com
} ${ }^{\dagger}$ k.luo@ucl.ac.uk use an orthogonal basis of central moments that relax to the equilibrium state of the continuous Maxwellian distribution [12]. Building on this work, many other efforts demonstrated that this model can largely enhance the stability of the LBM [12-31], even if it may lead to cumbersome analytical formulations and practical implementations, especially in three dimensions [32].

More recently, we revisited the adoption of central moments within the LBM framework [33-36] that differs from the cascaded scheme due to two main aspects: (i) the adoption of a nonorthogonal basis and (ii) the relaxation to the discrete second-order truncated equilibrium populations. The corresponding collision operator loses the above-mentioned pyramidal structure, entails a very intelligible algorithm, and shows excellent properties in terms of accuracy, convergence, and stability. More interestingly, the latter condition appears very natural, orthodox, and it is totally compliant with the BGK counterpart. This choice is not an approximation or an assumption, but it is formally derived through the adoption of a transformation matrix allowing us to go from pre-collision, equilibrium, and post-collision populations to (pre-collision, equilibrium, and post-collision) central moments, and vice versa $[33,34]$. As a consequence, we proved that it is possible to derive central-moments-based schemes for a broad range of governing equations, as preconditioning [37] and shallow water equations [38]. A compelling proof of the generality of the algorithm is provided in [39], where a central-moments-based scheme able to recover the solution of the incompressible Navier-Stokes equations for magnetohydrodynamics has been derived. If one compares the algorithm outlined in [39] with the nonconductive case [33], it is possible to appreciate that the structure of the algorithm is identical. The only difference lies in the equilibrium central moments, that are enriched 
by terms accounting for the magnetic field. This is totally consistent with the BGK counterpart, where equilibrium populations just account for an extra magnetic field-dependent term [40]. Moreover, a third-order velocity term has been added to the equilibrium to simulate jet breakup [41] and, again, it is found that it does not affect the algorithmic procedure. In principle, it is possible to derive a central-momentsbased procedure for whatever arbitrary truncation order of the equilibrium populations.

In this paper, we derive three-dimensional models for progressively more sophisticated equilibrium states. Consistent with the previous assessment, we find that the algorithm can be kept constant and the only changes apply to the equilibrium central moments. More interestingly, we find that equilibrium CMs collapse into those of the continuous Maxwellian distribution when a discrete sixth-order truncation distribution is considered, thus giving a theoretical $a$ priori backing for the key assumption behind the cascaded scheme. Some details are reported in the appendixes.

\section{LATTICE BOLTZMANN SCHEMES WITH HIGHER-ORDER HERMITE POLYNOMIALS}

In this section, the BGK LBM is briefly recalled. Then, theoretical derivations of the central-moments-based scheme are discussed.

\section{A. BGK models}

The Boltzmann equation describes the evolution of the probability distribution function $f(\boldsymbol{x}, \boldsymbol{\xi}, t)$ of finding a particle at the position $x$ and time $t$ moving with velocity $\xi$ :

$$
\partial_{t} f(\boldsymbol{x}, \xi, t)+\boldsymbol{\xi} \cdot \nabla f(\boldsymbol{x}, \boldsymbol{\xi}, t)=\Omega(f),
$$

where $\Omega$ is called a collision operator. The fluid density $\rho$ and velocity $\boldsymbol{u}$ can be obtained as the moments of the distribution, i.e.,

$$
\begin{aligned}
\rho & =\int f(\boldsymbol{x}, \boldsymbol{\xi}, t) d \boldsymbol{\xi}, \\
\rho \boldsymbol{u} & =\int f(\boldsymbol{x}, \boldsymbol{\xi}, t) \boldsymbol{\xi} d \boldsymbol{\xi} .
\end{aligned}
$$

By adopting the popular single-relaxation-time BGK model [7], the collision stage can be written as

$$
\Omega(f)=\omega\left(f^{(0)}(\rho, \boldsymbol{u})-f(\boldsymbol{x}, \boldsymbol{\xi}, t)\right),
$$

where $\omega$ is a relaxation frequency and $f^{(0)}$ is the local Maxwell-Boltzmann equilibrium distribution, that is,

$$
f^{(0)}=\frac{\rho(\boldsymbol{x}, t)}{(2 \pi)^{D / 2}} \exp \left(-\frac{(\boldsymbol{u}(\boldsymbol{x}, t)-\boldsymbol{\xi})^{2}}{2}\right),
$$

$D$ being the spatial dimension.

This equilibrium distribution can be expanded in Hermite polynomials up to an arbitrary order $N$, that is,

$$
f^{(0)^{N}}=w(\xi) \sum_{n=0}^{N} \frac{1}{n !} \mathcal{H}^{(n)}(\boldsymbol{\xi}): \boldsymbol{a}_{0}^{(n)},
$$

where $w(\xi)=\exp \left(\xi^{2} / 2\right)$ are the Gaussian weights associated with the Hermite polynomials $\mathcal{H}^{(n)}$ and $\boldsymbol{a}_{0}^{(n)}$ are the Hermite coefficients of order $n$ [42-44].
Let us consider an Eulerian basis $\boldsymbol{x}=[x, y, z]$. By discretizing the velocity space, Eq. (1) can be rewritten as

$$
\partial_{t} f_{i}+\boldsymbol{\xi}_{i} \cdot \nabla f_{i}=\omega\left(f_{i}^{(0)}(\rho, \boldsymbol{u})-f_{i}\right)
$$

where the index $i$ spans a finite set of direction. In this case, $i \in[0 \ldots 26]$ as the D3Q27 model is considered [2]. Following the works by Malaspinas [45] and Coreixas [46,47], the equilibrium state is expanded as follows:

$$
\begin{aligned}
f_{i}^{(0)}= & w_{i} \rho\left[1+\frac{\boldsymbol{\xi}_{i} \cdot \boldsymbol{u}}{c_{s}^{2}}+\frac{1}{2 c_{s}^{4}} \mathcal{H}_{i}^{(2)}: \boldsymbol{u} \boldsymbol{u}\right. \\
& +\frac{1}{2 c_{s}^{6}}\left(\mathcal{H}_{i x x y}^{(3)} u_{x}^{2} u_{y}+\mathcal{H}_{i x x z}^{(3)} u_{x}^{2} u_{z}+\mathcal{H}_{i x y y}^{(3)} u_{x} u_{y}^{2}\right. \\
& +\mathcal{H}_{i x z z}^{(3)} u_{x} u_{z}^{2}+\mathcal{H}_{i y z z}^{(3)} u_{y} u_{z}^{2}+\mathcal{H}_{i y y z}^{(3)} u_{y}^{2} u_{z} \\
& \left.+\mathcal{H}_{i x y z}^{(3)} u_{x} u_{y} u_{z}\right) \\
& +\frac{1}{4 c_{s}^{8}}\left(\mathcal{H}_{i x x y y}^{(4)} u_{x}^{2} u_{y}^{2}+\mathcal{H}_{i x x z z}^{(4)} u_{x}^{2} u_{z}^{2}+\mathcal{H}_{i y y z z}^{(4)} u_{y}^{2} u_{z}^{2}\right. \\
& \left.+2\left(\mathcal{H}_{i x y z z}^{(4)} u_{x} u_{y} u_{z}^{2}+\mathcal{H}_{i x y y z}^{(4)} u_{x} u_{y}^{2} u_{z}+\mathcal{H}_{i x x y z}^{(4)} u_{x}^{2} u_{y} u_{z}\right)\right) \\
& +\frac{1}{4 c_{s}^{10}}\left(\mathcal{H}_{i x x y z z}^{(5)} u_{x}^{2} u_{y} u_{z}^{2}+\mathcal{H}_{i x x y y z}^{(5)} u_{x}^{2} u_{y}^{2} u_{z}\right. \\
& \left.+\mathcal{H}_{i x y y z z}^{(5)} u_{x} u_{y}^{2} u_{z}^{2}\right) \\
& \left.+\frac{1}{8 c_{s}^{12}} \mathcal{H}_{i x x y y z z}^{(6)} u_{x}^{2} u_{y}^{2} u_{z}^{2}\right]
\end{aligned}
$$

where $c_{s}=1 / \sqrt{3}$ is the lattice sound speed and the velocity vector is written as $\boldsymbol{u}=\left[u_{x}, u_{y}, u_{z}\right]$. We refer to [46] for the complete expressions of the Hermite polynomials. By neglecting the terms $\mathcal{H}^{(3)}, \mathcal{H}^{(4)}, \mathcal{H}^{(5)}$, and $\mathcal{H}^{(6)}$, it is possible to recover the well-known second-order truncated equilibrium, i.e.,

$$
f_{i}^{(0)}=w_{i} \rho\left[1+\frac{\boldsymbol{\xi}_{i} \cdot \boldsymbol{u}}{c_{s}^{2}}+\frac{\left(\boldsymbol{\xi}_{i} \cdot \boldsymbol{u}\right)^{2}}{2 c_{s}^{4}}-\frac{\boldsymbol{u}^{2}}{2 c_{s}^{2}}\right] .
$$

\section{B. Central-moments-based models}

The crucial point to obtain a CM-based formulation is to shift the lattice directions by the local fluid velocity [12]:

$$
\begin{aligned}
\left|\bar{\xi}_{x i}\right\rangle & =\left|\xi_{x i}-u_{x}\right\rangle, \\
\left|\xi_{y i}\right\rangle & =\left|\xi_{y i}-u_{y}\right\rangle, \\
\left|\bar{\xi}_{z i}\right\rangle & =\left|\xi_{z i}-u_{z}\right\rangle .
\end{aligned}
$$

Lattice directions and weight coefficients are defined according to [34]. In order to move from the populations to moments, let us adopt the basis $\overline{\mathcal{T}}=\left[\bar{T}_{0}, \ldots, \bar{T}_{i}, \ldots, \bar{T}_{26}\right]$. Let us collect pre-collision, equilibrium, and post-collision CMs as

$$
\begin{aligned}
\left|k_{i}\right\rangle & =\left[k_{0}, \ldots, k_{i}, \ldots, k_{26}\right]^{\top}, \\
\left|k_{i}^{(0)}\right\rangle & =\left[k_{0}^{(0)}, \ldots, k_{i}^{(0)}, \ldots, k_{26}^{(0)}\right]^{\top}, \\
\left|k_{i}^{\star}\right\rangle & =\left[k_{0}^{\star}, \ldots, k_{i}^{\star}, \ldots, k_{26}^{\star}\right]^{\top},
\end{aligned}
$$

respectively. The first two quantities are evaluated by applying the transformation matrix $\overline{\mathcal{T}}$ to the corresponding distribution, 
that is,

$$
\left|k_{i}\right\rangle=\overline{\mathcal{T}}^{\top}\left|f_{i}\right\rangle, \quad\left|k_{i}^{(0)}\right\rangle=\overline{\mathcal{T}}^{\top}\left|f_{i}^{(0)}\right\rangle,
$$

where $\top$ denotes the transpose operator, $\left|f_{i}\right\rangle=$ $\left[f_{0}, \ldots f_{i}, \ldots f_{26}\right]^{\top}$, and $\left|f_{i}^{(0)}\right\rangle=\left[f_{0}^{(0)}, \ldots f_{i}^{(0)}, \ldots f_{26}^{(0)}\right]^{\top}$. By relaxing each moment with a proper frequency $\omega_{i}$, the collision of central moments takes place as

$$
k_{i}^{\star}=k_{i}+\omega_{i}\left(k_{i}^{(0)}-k_{i}\right)
$$

thus allowing us to reconstruct the post-collision populations, i.e.,

$$
\left|f_{i}^{\star}\right\rangle=\left(\overline{\mathcal{T}}^{\top}\right)^{-1}\left|k_{i}^{\star}\right\rangle
$$

that are eventually streamed.

One can immediately notice a feature of the above-outlined algorithm: It is totally independent from the expression of the equilibrium populations. In other words, one can choose whatever truncation order in Eq. (7) and it will always be possible to apply this procedure.

Now, let us investigate the values assumed by $\left|k_{i}^{(0)}\right\rangle$ for different truncation orders. By assuming the classical choice of $N=2$, we obtain the results in [34]:

$$
\begin{aligned}
& k_{0}^{(0)}=k_{9}^{(0)}=\rho, \quad k_{10}^{(0)}=-\rho u_{x}\left(u_{y}^{2}+u_{z}^{2}\right), \\
& k_{11}^{(0)}=-\rho u_{y}\left(u_{x}^{2}+u_{z}^{2}\right), \quad k_{12}^{(0)}=-\rho u_{z}\left(u_{x}^{2}+u_{y}^{2}\right), \\
& k_{13}^{(0)}=-\rho u_{x}\left(u_{y}^{2}-u_{z}^{2}\right), \quad k_{14}^{(0)}=-\rho u_{y}\left(u_{x}^{2}-u_{z}^{2}\right), \\
& k_{15}^{(0)}=-\rho u_{z}\left(u_{x}^{2}-u_{y}^{2}\right), \quad k_{16}^{(0)}=-\rho u_{x} u_{y} u_{z}, \\
& k_{17}^{(0)}=\rho\left(c_{s}^{2}+3 u_{x}^{2} u_{y}^{2}+3 u_{x}^{2} u_{z}^{2}+3 u_{y}^{2} u_{z}^{2}\right), \\
& k_{18}^{(0)}=\rho\left(c_{s}^{4}+3 u_{x}^{2} u_{y}^{2}+3 u_{x}^{2} u_{z}^{2}-3 u_{y}^{2} u_{z}^{2}\right), \\
& k_{19}^{(0)}=3 \rho u_{x}^{2}\left(u_{y}^{2}-u_{z}^{2}\right), \quad k_{20}^{(0)}=3 \rho u_{x}^{2} u_{y} u_{z}, \\
& k_{21}^{(0)}=3 \rho u_{x} u_{y}^{2} u_{z}, \quad k_{22}^{(0)}=3 \rho u_{x} u_{y} u_{z}^{2}, \\
& k_{23}^{(0)}=-\frac{\rho}{3} u_{x}\left(18 u_{y}^{2} u_{z}^{2}+u_{y}^{2}+u_{z}^{2}\right), \\
& k_{24}^{(0)}=-\frac{\rho}{3} u_{y}\left(18 u_{x}^{2} u_{z}^{2}+u_{x}^{2}+u_{z}^{2}\right), \\
& k_{25}^{(0)}=-\frac{\rho}{3} u_{z}\left(18 u_{x}^{2} u_{y}^{2}+u_{x}^{2}+u_{y}^{2}\right), \\
& k_{26}^{(0)}=\rho\left(c_{s}^{6}+10 u_{x}^{2} u_{y}^{2} u_{z}^{2}+u_{x}^{2} u_{y}^{2}+u_{x}^{2} u_{z}^{2}+u_{y}^{2} u_{z}^{2}\right),
\end{aligned}
$$

and $k_{1 \ldots 8}^{(0)}=0$. In this case, the determination of the postcollision populations requires the computation of 19 moments. Now, let us consider the presence of $\mathcal{H}^{(3)}$ in Eq. (7).
Equilibrium CMs read as follows:

$$
\begin{aligned}
& k_{0}^{(0)}=k_{9}^{(0)}=\rho, \quad k_{17}^{(0)}=\rho\left(c_{s}^{2}-u_{x}^{2} u_{y}^{2}-u_{x}^{2} u_{z}^{2}-u_{y}^{2} u_{z}^{2}\right), \\
& k_{18}^{(0)}=\rho\left(c_{s}^{4}-9 u_{x}^{2} u_{y}^{2}-9 u_{x}^{2} u_{z}^{2}+9 u_{y}^{2} u_{z}^{2}\right), \\
& k_{19}^{(0)}=-\rho u_{x}^{2}\left(u_{y}^{2}-u_{z}^{2}\right), \quad k_{20}^{(0)}=-\rho u_{x}^{2} u_{y} u_{z}, \\
& k_{21}^{(0)}=-\rho u_{x} u_{y}^{2} u_{z}, \quad k_{22}^{(0)}=-\rho u_{x} u_{y} u_{z}^{2}, \\
& k_{23}^{(0)}=4 \rho u_{x} u_{y}^{2} u_{z}^{2} \quad k_{24}^{(0)}=4 \rho u_{x}^{2} u_{y} u_{z}^{2}, \quad k_{25}^{(0)}=4 \rho u_{x}^{2} u_{y}^{2} u_{z}, \\
& k_{26}^{(0)}=\rho\left(c_{s}^{6}-10 u_{x}^{2} u_{y}^{2} u_{z}^{2}-9 u_{x}^{2} u_{y}^{2}-9 u_{x}^{2} u_{z}^{2}-9 u_{y}^{2} u_{z}^{2}\right),
\end{aligned}
$$

with $k_{1 \ldots 8}^{(0)}=k_{10 \ldots 16}^{(0)}=0$. With respect to the previous case, purely second-order terms vanish and only 12 moments are involved. Let us proceed by adding $\mathcal{H}^{(4)}$ :

$$
\begin{aligned}
& k_{0}^{(0)}=k_{9}^{(0)}=\rho, \quad k_{17}^{(0)}=\rho c_{s}^{2}, \\
& k_{18}^{(0)}=\rho c_{s}^{4}, \quad k_{23}^{(0)}=-\rho u_{x} u_{y}^{2} u_{z}^{2}, \\
& k_{24}^{(0)}=-\rho u_{x}^{2} u_{y} u_{z}^{2}, \quad k_{25}^{(0)}=-\rho u_{x}^{2} u_{y}^{2} u_{z}, \\
& k_{26}^{(0)}=\rho\left(c_{s}^{6}+5 u_{x}^{2} u_{y}^{2} u_{z}^{2}\right),
\end{aligned}
$$

with $k_{1 \ldots 8}^{(0)}=k_{10 \ldots 16}^{(0)}=k_{19 \ldots 22}^{(0)}=0$, and $\mathcal{H}^{(5)}$

$$
\begin{aligned}
& k_{0}^{(0)}=k_{9}^{(0)}=\rho, \quad k_{17}^{(0)}=\rho c_{s}^{2}, \\
& k_{18}^{(0)}=\rho c_{s}^{4}, \quad k_{26}^{(0)}=\rho\left(c_{s}^{6}-u_{x}^{2} u_{y}^{2} u_{z}^{2}\right),
\end{aligned}
$$

with $k_{1 \ldots 8}^{(0)}=k_{10 \ldots 16}^{(0)}=k_{19 \ldots 25}^{(0)}=0$. One can immediately observe that an Hermite polynomial of order $N$ promotes the annihilation of velocity terms of the same order. Finally, we consider $\mathcal{H}^{(6)}$ too, i.e.,

$$
\begin{aligned}
& k_{0}^{(0)}=k_{9}^{(0)}=\rho, \quad k_{17}^{(0)}=\rho c_{s}^{2}, \\
& k_{18}^{(0)}=\rho c_{s}^{4}, \quad k_{26}^{(0)}=\rho c_{s}^{6} .
\end{aligned}
$$

Interestingly, we notice that the values of the equilibrium CMs obtained by considering equilibrium populations with Hermite polynomials up to the sixth order leads to the values used in the cascaded LBM. In this way, we have formally demonstrated that the relaxation to the equilibrium of the continuous Maxwellian distribution is not only an assumption to be justified a posteriori with an enhanced Galilean invariance. In fact, it can be interpreted as the relaxation to the discrete distribution accounting for Hermite polynomials up to the sixth order. Moreover, the outlined procedure is totally compliant with the BGK counterpart. In fact, if one chooses to adopt equilibrium CMs corresponding to Hermite polynomials up to order $N$ and relaxes all the moments with a common unique rate, the BGK scheme with equilibrium populations $f_{i}^{(0)}$ accounting for Hermite polynomials up to the same order is exactly recovered. In addition, the method is consistent with the classical multiple-relaxation time, too, $[24,25,37]$, where raw (and not central) moments are considered [9]. The transformation matrix $\bar{T}$ can be decomposed as $\bar{T}=T+S(\boldsymbol{u})$, where $T$ is obtained by considering $\xi$ instead of $\bar{\xi}$ in Eq. (A1) and $S(\boldsymbol{u})$ is a shift matrix [37]. Therefore, the MRT is simply recovered from the present central-momentsbased formulation without performing any shift of the lattice directions [or by imposing $S(\boldsymbol{u})=0)$ ]. Notice that the idea of 
TABLE I. Linear stability analysis: maximum eigenvalue of the matrix $\boldsymbol{\Theta}$ for different values of the relaxation frequency and Mach number $\left(\mathrm{Ma}=\tilde{u} / c_{s}\right)$ by using Hermite polynomials up to the second order and sixth order. It is possible to appreciate that eigenvalues are always smaller than 1 in the latter scenario.

\begin{tabular}{|c|c|c|c|c|c|c|c|c|c|c|c|c|c|c|}
\hline \multirow[b]{2}{*}{$\omega_{v}$} & \multicolumn{2}{|c|}{0} & \multicolumn{2}{|c|}{0.1732} & \multicolumn{2}{|c|}{0.3464} & \multicolumn{2}{|c|}{0.5196} & \multicolumn{2}{|c|}{0.6928} & \multicolumn{2}{|c|}{0.8660} & \multicolumn{2}{|c|}{1.0392} \\
\hline & $\mathcal{H}_{i}^{(2)}$ & $\mathcal{H}_{i}^{(6)}$ & $\mathcal{H}_{i}^{(2)}$ & $\mathcal{H}_{i}^{(6)}$ & $\mathcal{H}_{i}^{(2)}$ & $\mathcal{H}_{i}^{(6)}$ & $\mathcal{H}_{i}^{(2)}$ & $\mathcal{H}_{i}^{(6)}$ & $\mathcal{H}_{i}^{(2)}$ & $\mathcal{H}_{i}^{(6)}$ & $\mathcal{H}_{i}^{(2)}$ & $\mathcal{H}_{i}^{(6)}$ & $\mathcal{H}_{i}^{(2)}$ & $\mathcal{H}_{i}^{(6)}$ \\
\hline 1.90 & 0.9954 & 0.9954 & 0.9979 & 0.9982 & 0.9982 & 0.9994 & 0.9996 & 0.9998 & 1.0387 & 0.9999 & 1.0819 & 0.9999 & 1.1803 & 0.9999 \\
\hline 1.92 & 0.9954 & 0.9954 & 0.9979 & 0.9982 & 0.9982 & 0.9994 & 0.9996 & 0.9998 & 1.0393 & 0.9999 & 1.0831 & 0.9999 & 1.1818 & 0.9999 \\
\hline 1.94 & 0.9954 & 0.9954 & 0.9979 & 0.9982 & 0.9982 & 0.9994 & 0.9996 & 0.9998 & 1.0400 & 0.9999 & 1.0843 & 0.9999 & 1.1833 & 0.9999 \\
\hline 1.96 & 0.9954 & 0.9954 & 0.9979 & 0.9982 & 0.9982 & 0.9994 & 0.9996 & 0.9998 & 1.0407 & 0.9999 & 1.0855 & 0.9999 & 1.1849 & 0.9999 \\
\hline 1.98 & 0.9954 & 0.9954 & 0.9979 & 0.9982 & 0.9982 & 0.9994 & 0.9996 & 0.9998 & 1.0414 & 0.9999 & 1.0867 & 0.9999 & 1.1864 & 0.9999 \\
\hline 2.00 & 0.9954 & 0.9954 & 0.9979 & 0.9982 & 0.9982 & 0.9994 & 0.9996 & 0.9998 & 1.0421 & 0.9999 & 1.0879 & 0.9999 & 1.1879 & 0.9999 \\
\hline
\end{tabular}

a shift matrix for passing from the frame at rest to the one co-moving with the fluid was first introduced by Asinari [15] within the cascaded LBM.

In the Supplemental Material, a script [48] allows the reader to perform the symbolic manipulations to obtain all the involved quantities.

The discrete LB equation introduces an error that is proportional to the spatial scale and time step. Moreover, the method is explicit in time and it is based on polynomial approximations of the continuous Boltzmann equation. Given all these features, the LBM is prone to numerical instabilities. in order to investigated its behavior, a von Neumann linear stability analysis is performed [49-52] and the approach devised in $[47,53]$ is followed. In short, the collide-and-stream algorithm is linearized around a mean flow through the injection of a perturbation. By evaluating the growth rate of the perturbation, it is possible to assess if it will increase or decrease depending on the wave number, the Mach number, and the relaxation frequency. Specifically, one has to compute the eigenvalues $\left|\theta_{i}\right\rangle$ of the matrix,

$$
\boldsymbol{\Theta}=\mathbf{E}(\mathbf{I}+\mathbf{J}),
$$

where $E_{i j}=e^{-i \xi_{i} \cdot \mathbf{l}}$ (with $i=\sqrt{-1}$ ), $\mathbf{I}$ is the unit tensor and $\mathbf{I}=\left[l_{x}, l_{y}, l_{z}\right]$ is the wave number. The components of the Jacobian matrix $\mathbf{J}$ are computed as

$$
J_{i j}=\left.\frac{\partial \Omega_{i}}{\partial \tilde{f}_{j}}\right|_{\tilde{f}_{j}},
$$

where $j \in[0 \ldots 26], \tilde{f}_{j}=\left.f_{j}^{(0)}\right|_{\tilde{\rho}, \tilde{u}}$ is a distribution function related to an equilibrium state given by the set of variables $\tilde{\rho}$ and $\tilde{\boldsymbol{u}}$. Our central-moments-based collision operator $\left|\Omega_{i}\right\rangle$ can be synthetically written as

$$
\left|\Omega_{i}\right\rangle=\left(\overline{\mathcal{T}}^{\top}\right)^{-1} \boldsymbol{\Lambda} \overline{\mathcal{T}}^{\top}\left(\left|f_{i}\right\rangle-\left|f_{i}^{(0)}\right\rangle\right),
$$

where $\boldsymbol{\Lambda}=\operatorname{diag}\left[1,1,1,1, \omega_{\nu}, \omega_{\nu}, \omega_{\nu}, \omega_{\nu}, \omega_{\nu}, 1, \ldots, 1\right]$ is a $(27 \times 27)$ relaxation matrix with $\omega_{v}=\left(\frac{v}{c_{s}^{2}}+\frac{1}{2}\right)^{-1}, v$ being the fluid kinematic viscosity.

For given values of $\tilde{\rho}$ and $\tilde{\boldsymbol{u}}$, the von Neumann stability criterion assesses that the LBE is stable if the complex modulus of all the eigenvalues $\left|\theta_{i}\right\rangle$ is smaller than 1 for all values of $\mathbf{l}$, whose components range from 0 to $2 \pi$. Let us set $\tilde{\rho}=1$ and $\tilde{\boldsymbol{u}}=[\tilde{u}, \tilde{u}, \tilde{u}]$. Notice that the resultant flow field is not aligned with the Cartesian grid, thus leading to a more challenging setting $[47,53]$. By varying the relaxation frequency as $\omega_{\nu} \in[1.9: 2]$, we compute the eigenvalues of $\Theta$ by using the LAPACK ++ (linear algebra package for the $\mathrm{C}++$ language) and the maximum complex modulus $\theta_{\max }=$ $\max _{i}\left[\sqrt{\operatorname{real}\left(\theta_{i}\right)^{2}+\operatorname{imag}\left(\theta_{i}\right)^{2}}\right]$ is reported for progressively larger values of $\tilde{u}$ in Table I. The von Neumann analysis shows that the algorithm becomes unstable if $\tilde{u}>0.4$ if only $\mathcal{H}_{i}^{(2)}$ is considered. Conversely, $\theta_{\max }<1$ if Hermite polynomials up to the sixth order are accounted for. This is in line with previous results obtained with the D2Q9 lattice for both the BGK and regularized collision model [47]. Notice that findings in Table I correspond to $l_{x}=l_{y}=l_{z}=0$, that is found to be the most critical scenario for the given flow conditions. However, we span the whole spectrum and combination of values of the wave number and we found always that $\theta_{\max }<1$ if equilibrium populations include $\mathcal{H}_{i}^{(6)}$. In order to help the reader

TABLE II. Linear stability analysis: maximum eigenvalue of the matrix $\boldsymbol{\Theta}$ for different values of the relaxation frequency and the three

\begin{tabular}{|c|c|c|c|c|c|c|c|c|c|}
\hline \multirow[b]{2}{*}{$\underline{\omega_{v}}$} & \multicolumn{3}{|c|}{0.6928} & \multicolumn{3}{|c|}{0.8660} & \multicolumn{3}{|c|}{1.0392} \\
\hline & $\mathcal{H}_{i}^{(3)}$ & $\mathcal{H}_{i}^{(4)}$ & $\mathcal{H}_{i}^{(5)}$ & $\mathcal{H}_{i}^{(3)}$ & $\mathcal{H}_{i}^{(4)}$ & $\mathcal{H}_{i}^{(5)}$ & $\mathcal{H}_{i}^{(3)}$ & $\mathcal{H}_{i}^{(4)}$ & $\mathcal{H}_{i}^{(5)}$ \\
\hline 1.90 & 1.0095 & 1.0029 & 1.0004 & 1.0338 & 1.0106 & 1.0020 & 1.2207 & 1.0253 & 1.0254 \\
\hline 1.92 & 1.0096 & 1.0029 & 1.0004 & 1.0336 & 1.0106 & 1.0020 & 1.2205 & 1.0253 & 1.0254 \\
\hline 1.94 & 1.0096 & 1.0029 & 1.0004 & 1.0335 & 1.0106 & 1.0020 & 1.2203 & 1.0253 & 1.0257 \\
\hline 1.96 & 1.0096 & 1.0029 & 1.0004 & 1.0333 & 1.0106 & 1.0020 & 1.2200 & 1.0253 & 1.0259 \\
\hline 1.98 & 1.0096 & 1.0029 & 1.0004 & 1.0332 & 1.0106 & 1.0020 & 1.2197 & 1.0252 & 1.0260 \\
\hline 2.00 & 1.0096 & 1.0029 & 1.0004 & 1.0330 & 1.0106 & 1.0020 & 1.2193 & 1.0252 & 1.0261 \\
\hline
\end{tabular}
largest Mach numbers by using third-, fourth- and fifth-order Hermite polynomials. As $\theta_{\max }>1$, Hermite polynomials lower than the sixth order do not successfully contribute to the stability improvement. 


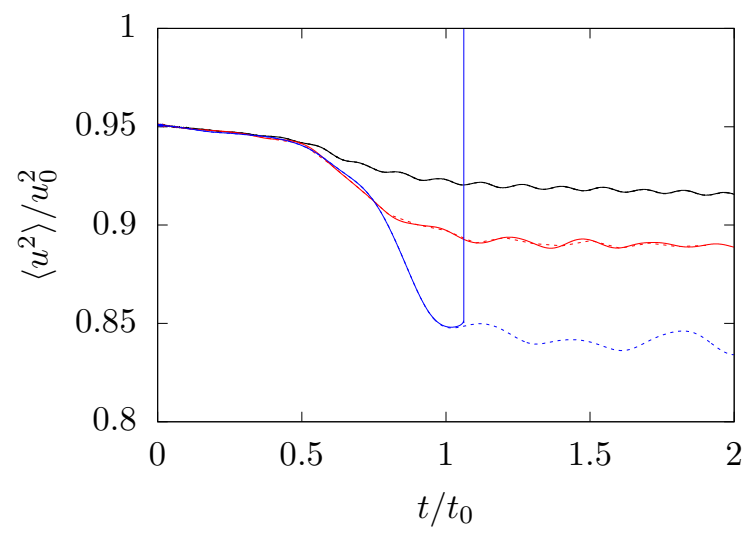

FIG. 1. Double shear layer: time evolution of the normalized mean energy at $\mathrm{Ma}=0.2$ (black), 0.35 (red), and 0.57 (blue) by $\mathcal{P}_{1}$ (continuous line) and $\mathcal{P}_{2}$ (dashed line). The difference between the two grows with Ma. For the largest value of the Mach number, only the adoption of higher-order polynomials can lead to a stable run. Please refer to the online version of this paper to see this figure in colour.

investigate the stability of the above-outlined algorithm, we attach a script [48] allows the reader to perform the symbolic manipulations to obtain all the involved quantities. to perform the von Neumann analysis. Finally, we investigate the incidence of each order on the stability properties. Specifically, we repeat the experiments characterized by $\tilde{u}=0.4,0.5,0.6$ (i.e., those where $\mathcal{H}_{i}^{(2)}$ fails to guarantee the stability) by progressively enriching the equilibrium distribution functions. The corresponding maximum eigenvalues are reported in Table II. Even if $\theta_{\max }$ appears to be reduced, its values are still larger than 1 . Therefore, we conclude that the adoption of the full set of Hermite polynomials plays a pivotal role in stabilizing the procedure.

\section{NUMERICAL EXPERIMENTS}

We compare the adoption of Eqs. (14) and (18) against the double shear layer [54], that is widely acknowledged as an excellent candidate to evaluate the stability of numerical schemes $[46,55,56]$. Let us consider a doubly periodic domain with $(x, y) \in[0, L]^{2}$. The initial flow field is composed of two longitudinal shear layers and a transverse perturbation is superimposed, i.e.,

$$
u_{x}= \begin{cases}u_{0} \tanh \left[\kappa\left(\frac{y}{L}-\frac{1}{4}\right)\right], & \frac{y}{L} \leqslant \frac{1}{2}, \\ u_{0} \tanh \left[\kappa\left(\frac{3}{4}-\frac{y}{L}\right)\right], & \frac{y}{L}>\frac{1}{2},\end{cases}
$$

and

$$
u_{y}=u_{0} \delta \sin \left[2 \pi\left(\frac{x}{L}+\frac{1}{4}\right)\right],
$$

where $\kappa=80$ and $\delta=0.05$. Fluid dynamics manifests the roll-up of the shear layers and the generation of two counterrotating vortices due to a Kelvin-Helmotz instability mechanism. The Reynolds number is $\operatorname{Re}=u_{0} L / v=3 \times 10^{4}$. We run simulations by setting $L=256$ and accounting for secondand sixth-order Hermite polynomials and these are labeled as $\mathcal{P}_{1}$ and $\mathcal{P}_{2}$, respectively. Moreover, three values of the
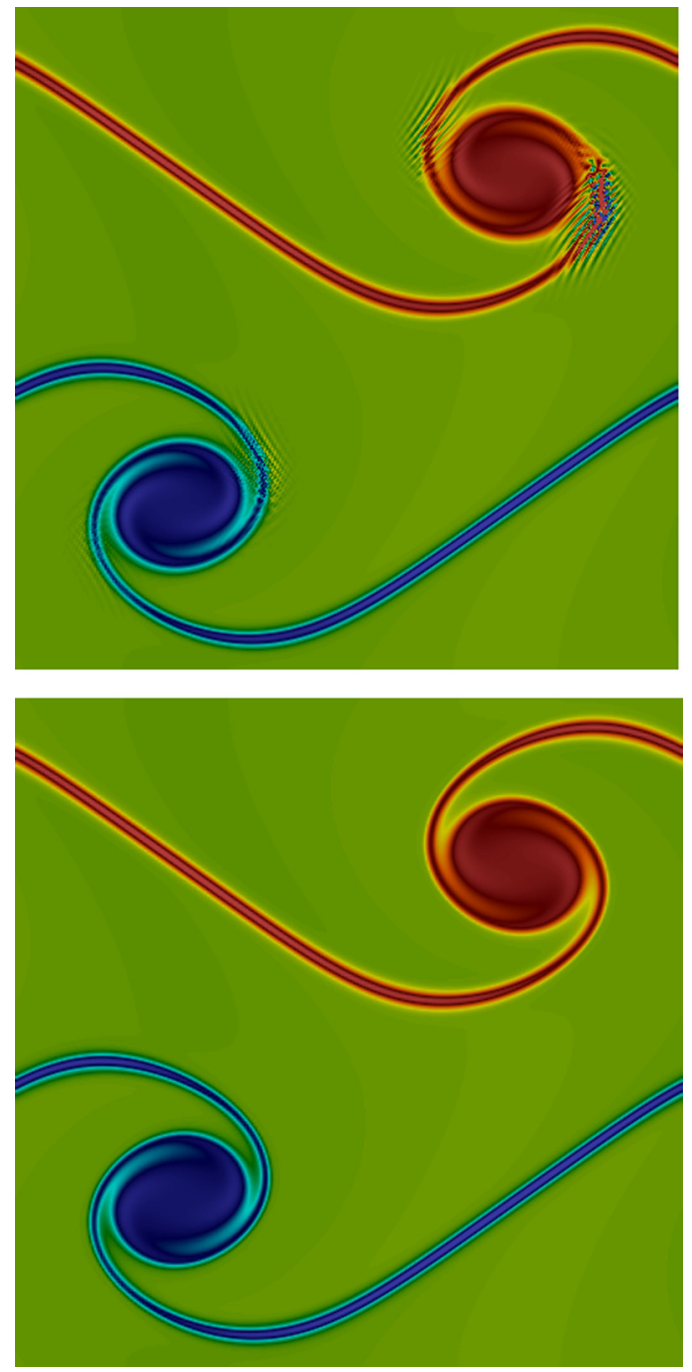

FIG. 2. Double shear layer: contour plot of the vorticity by $\mathcal{P}_{1}$ (left) and $\mathcal{P}_{2}$ (right) at $\mathrm{Ma}=0.57$ and $t=1.062 t_{0}$. Map ranges from -0.05 (blue) to 0.05 (red). Spurious artifacts arise when only $\mathcal{H}^{(2)}$ is considered, whereas the presence of terms up to $\mathcal{H}^{(6)}$ generates a smooth solution. Please refer to the online version of this paper to see this figure in colour.

Mach number are considered, i.e., $\mathrm{Ma}=u_{0} / c_{s}=0.2,0.35$, and 0.57 [46]. In Fig. 1, the time histories of the mean kinetic energy (normalized by $u_{0}$ ) are depicted. For the lowest values of Ma, solutions provided $\mathcal{P}_{1}$ and $\mathcal{P}_{2}$ are substantially overlapped. A slight mismatch between the two arises at $\mathrm{Ma}=0.35$. In fact, terms related to higher-velocity power now becomes non-negligible. More interestingly, the beneficial effects played by higher-order Hermite polynomials can be appreciated in the scenario where $\mathrm{Ma}=0.57$ is considered. In fact, $\mathcal{P}_{1}$ becomes unstable at $t \approx 1.062 t_{0}$ (with $t_{0}=L / u_{0}$ ). Conversely, the run corresponding to the adoption of Hermite polynomials up to the sixth order can run to $t=2 t_{0}$ without facing any issue.

Further insights are provided in Fig. 2, where the vorticity is sketched for $\mathcal{P}_{1}$ and $\mathcal{P}_{2}$ at $\mathrm{Ma}=0.57$ in correspondence to the time instant when the former blows up. When considering only Hermite polynomials up to the second order, spurious 


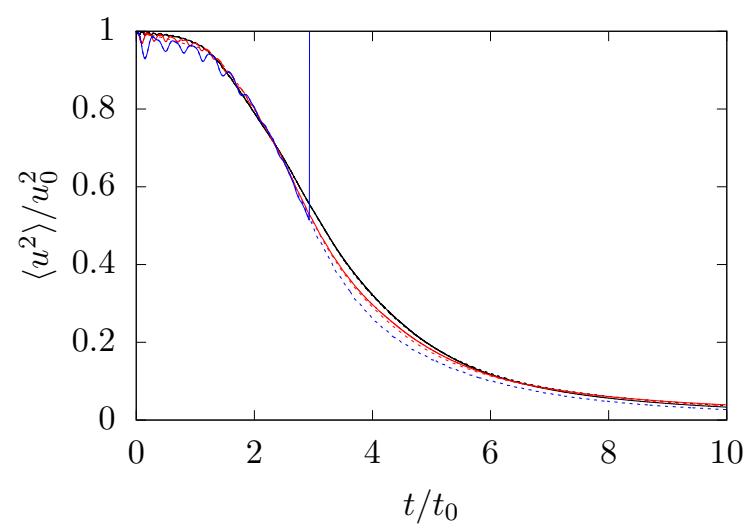

FIG. 3. Taylor-Green vortex: time evolution of the normalized mean energy at $\mathrm{Ma}=0.2$ (black), 0.4 (red), and 0.64 (blue) by $\mathcal{P}_{1}$ (continuous line) and $\mathcal{P}_{2}$ (dashed line). Using sixth-order Hermite polynomials allows us to perform successfully the highest-Mach run. Please refer to the online version of this paper to see this figure in colour.

artifacts develop in the flow field near the zones of larger velocities, thus contaminating the solution and even leading to a sudden crash of the simulation. Conversely, $\mathcal{P}_{2}$ does not show such deleterious effects. In other words, the adoption of higher-order polynomials plays a positive role in the numerical scheme, allowing stable simulations at a higher Mach number.

For the sake of completeness, we investigate the numerical performance of the proposed methodology against a threedimensional Taylor-Green vortex [57,58]. Let us consider a cubic periodic domain with edge length $D$. The flow develops due to the following initial conditions:

$$
\begin{gathered}
u_{x}(\boldsymbol{x}, t=0)=U_{0} \cos x \sin y \sin z, \\
u_{y}(\boldsymbol{x}, t=0)=\frac{U_{0}}{2} \sin x \cos y \sin z, \\
u_{z}(\boldsymbol{x}, t=0)=-\frac{U_{0}}{2} \sin x \sin y \cos z .
\end{gathered}
$$

By setting $D=128$ and $\operatorname{Re}=10^{4}$ (with $\operatorname{Re}=U_{0} D / v$ ), we run simulations at different Mach number, i.e., $\mathrm{Ma}=U_{0} / c_{s}=$ 0.2, 0.4 and 0.64.In Fig. 3, the time evolution of the normalized kinetic energy is reported for the above-mentioned values of Ma by adopting Hermite polynomials up to the second and sixth order. For the highest value of $\mathrm{Ma}, \mathcal{P}_{1}$ becomes unstable at $t=2.941 t_{0}$ (with $t_{0}=D / U_{0}$ ). Conversely, the adoption of sixth-order polynomials confirms its beneficial incidence as it allows us to simulate the whole desired time span. The vorticity field at salient time instants is sketched in Fig. 4, where it is demonstrated that the adopted algorithm is able to capture fine flow features and strong local gradients. In the Supplemental Material, an animation shows the evolution of the vorticity field [48] allows the reader to perform the symbolic manipulations to obtain all the involved quantities.

\section{CONCLUSIONS}

Building on our central-moments-based lattice Boltzmann scheme, we demonstrate that it is possible to relax moments
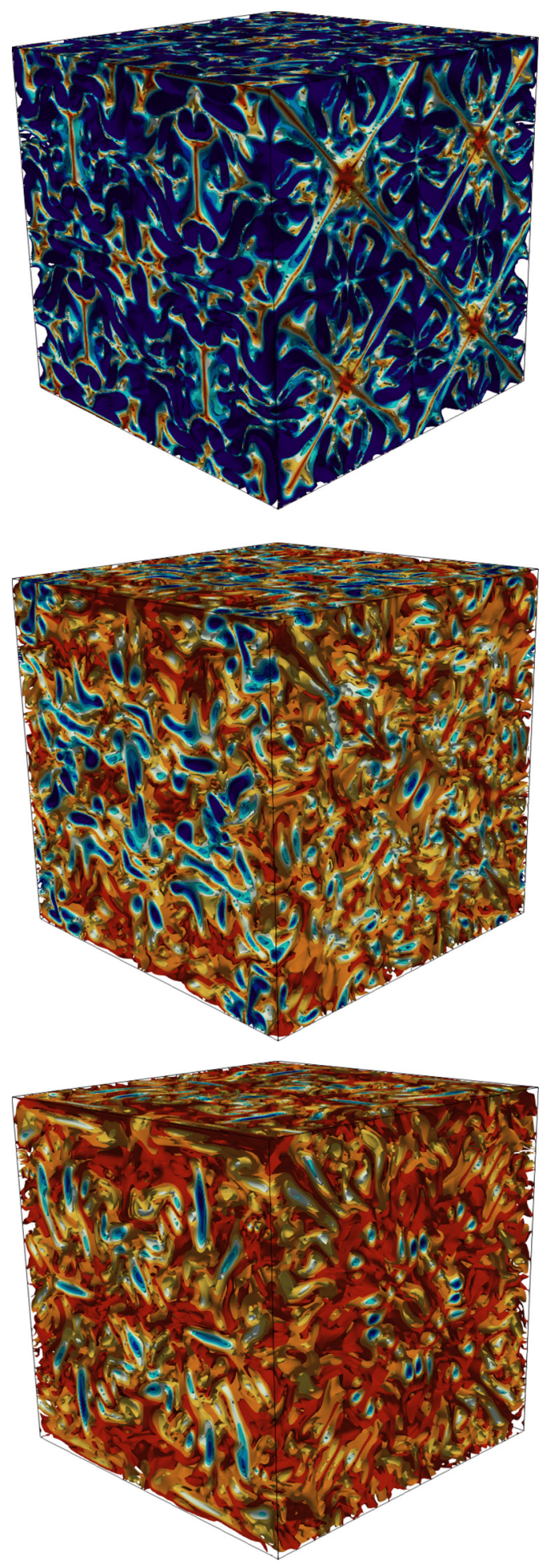

FIG. 4. Three-dimensional Taylor-Green vortex at $\mathrm{Ma}=0.64$. Contour plot of the vorticity by $\mathcal{P}_{2}$ (right) at salient time instants: $t=2 t_{0}$ (top panel), $7 t_{0}$ (middle panel), and $9 t_{0}$ (bottom panel). Please refer to the online version of this paper to see this figure in colour.

to an equilibrium state accounting for any Hermite polynomial up to whatever order $N$. Moreover, we provide an a priori theoretical proof of a common assumption of the cascaded LBM, that is, the equivalence of the relaxation to the equilibrium 
of the discrete and continuous Maxwellian distributions. In particular, one can observe the following:

(i) The adoption of a Hermite polynomial of order $N$ leads to the annihilation of velocity terms of the same order.

(ii) When the highest order is considered, equilibrium CMs assume the values corresponding to the continuous Maxwellian distribution, thus corroborating the assumption by several previous efforts.

(iii) The methodology is totally compliant with the BGK counterpart of order $N$.

(iv) The MRT LBM can be interpreted as a particular case obtained by building the transformation matrix with $\xi$ instead of $\bar{\xi}$.

\section{ACKNOWLEDGMENTS}

This article is based upon work from COST Action MP1305, supported by COST (European Cooperation in Science and Technology). A.D.R would like to thank Dr. Christophe Coreixas and Mr. Linlin Fei for insightful discussions and very valuable suggestions. K.H.L. would like to acknowledge funding from the UK Engineering and Physical Sciences Research Council under the projects "UK Consortium on Mesoscale Engineering Sciences (UKCOMES)" (Grants No. EP/L00030X/1 and No. EP/R029598/1). The authors are also grateful to the anonymous referees, who helped to improve the manuscript with their comments.

\section{APPENDIX A: D3Q27 MODEL: \\ TRANSFORMATION MATRIX}

The components of the matrix $\overline{\mathcal{T}}$ read as follows:

$$
\begin{aligned}
\left|\bar{T}_{0}\right\rangle & =|1, \ldots, 1\rangle, \quad\left|\bar{T}_{1}\right\rangle=\left|\bar{\xi}_{x i}\right\rangle, \quad\left|\bar{T}_{2}\right\rangle=\left|\bar{\xi}_{y i}\right\rangle, \\
\left|\bar{T}_{3}\right\rangle & =\left|\bar{\xi}_{z i}\right\rangle, \quad\left|\bar{T}_{4}\right\rangle=\left|\bar{\xi}_{x i} \bar{\xi}_{y i}\right\rangle, \quad\left|\bar{T}_{5}\right\rangle=\left|\bar{\xi}_{x i} \bar{\xi}_{z i}\right\rangle, \\
\left|\bar{T}_{6}\right\rangle & =\left|\bar{\xi}_{y i} \bar{\xi}_{z i}\right\rangle, \quad\left|\bar{T}_{7}\right\rangle=\left|\bar{\xi}_{x i}^{2}-\bar{\xi}_{y i}^{2}\right\rangle, \quad\left|\bar{T}_{8}\right\rangle=\left|\bar{\xi}_{x i}^{2}-\bar{\xi}_{z i}^{2}\right\rangle, \\
\left|\bar{T}_{9}\right\rangle & =\left|\bar{\xi}_{x i}^{2}+\bar{\xi}_{y i}^{2}+\bar{\xi}_{z i}^{2}\right\rangle, \quad\left|\bar{T}_{10}\right\rangle=\left|\bar{\xi}_{x i} \bar{\xi}_{y i}^{2}+\bar{\xi}_{x i} \bar{\xi}_{z i}^{2}\right\rangle, \\
\left|\bar{T}_{11}\right\rangle & =\left|\bar{\xi}_{x i}^{2} \bar{\xi}_{y i}+\bar{\xi}_{y i} \bar{\xi}_{z i}^{2}\right\rangle, \quad\left|\bar{T}_{12}\right\rangle=\left|\bar{\xi}_{x i}^{2} \bar{\xi}_{z i}+\bar{\xi}_{y i}^{2} \bar{\xi}_{z i}\right\rangle \\
\left|\bar{T}_{13}\right\rangle & =\left|\bar{\xi}_{x i} \bar{\xi}_{y i}^{2}-\bar{\xi}_{x i} \bar{\xi}_{z i}^{2}\right\rangle, \quad\left|\bar{T}_{14}\right\rangle=\left|\bar{\xi}_{x i}^{2} \bar{\xi}_{y i}-\bar{\xi}_{y i} \bar{\xi}_{z i}^{2}\right\rangle \\
\left|\bar{T}_{15}\right\rangle & =\left|\bar{\xi}_{x i}^{2} \bar{\xi}_{z i}-\bar{\xi}_{y i}^{2} \bar{\xi}_{z i}\right\rangle, \quad\left|\bar{T}_{16}\right\rangle=\left|\bar{\xi}_{x i} \bar{\xi}_{y i} \bar{\xi}_{z i}\right\rangle, \\
\left|\bar{T}_{17}\right\rangle & =\left|\bar{\xi}_{x i}^{2} \bar{\xi}_{y i}^{2}+\bar{\xi}_{x i}^{2} \bar{\xi}_{z i}^{2}+\bar{\xi}_{y i}^{2} \bar{\xi}_{z i}^{2}\right\rangle, \\
\left|\bar{T}_{18}\right\rangle & =\left|\bar{\xi}_{x i}^{2} \bar{\xi}_{y i}^{2}+\bar{\xi}_{x i}^{2} \bar{\xi}_{z i}^{2}-\bar{\xi}_{y i}^{2} \bar{\xi}_{z i}^{2}\right\rangle, \\
\left|\bar{T}_{19}\right\rangle & =\left|\bar{\xi}_{x i}^{2} \bar{\xi}_{y i}^{2}-\bar{\xi}_{x i}^{2} \bar{\xi}_{z i}^{2}\right\rangle, \quad\left|\bar{T}_{20}\right\rangle=\left|\bar{\xi}_{x i}^{2} \bar{\xi}_{y i} \bar{\xi}_{z i}\right\rangle, \\
\left|\bar{T}_{21}\right\rangle & =\left|\bar{\xi}_{x i} \bar{\xi}_{y i}^{2} \bar{\xi}_{z i}\right\rangle, \quad\left|\bar{T}_{22}\right\rangle=\left|\bar{\xi}_{x i} \bar{\xi}_{y i} \bar{\xi}_{z i}^{2}\right\rangle \\
\left|\bar{T}_{23}\right\rangle & =\left|\bar{\xi}_{x i} \bar{\xi}_{y i}^{2} \bar{\xi}_{z i}^{2}\right\rangle, \quad\left|\bar{T}_{24}\right\rangle=\left|\bar{\xi}_{x i}^{2} \bar{\xi}_{y i} \bar{\xi}_{z i}^{2}\right\rangle \\
\left|\bar{T}_{25}\right\rangle & =\left|\bar{\xi}_{x i}^{2} \bar{\xi}_{y i}^{2} \bar{\xi}_{z i}\right\rangle, \quad\left|\bar{T}_{26}\right\rangle=\left|\bar{\xi}_{x i}^{2} \bar{\xi}_{y i}^{2} \bar{\xi}_{z i}^{2}\right\rangle
\end{aligned}
$$

\section{APPENDIX B: D2Q9 MODEL}

We prove that the methodology can be applied to the two-dimensional D2Q9 lattice velocity space. Let us consider an Eulerian basis $\boldsymbol{x}=[x, y]$ and a velocity vector $\boldsymbol{u}=\left[u_{x}, u_{y}\right]$. Lattice directions are $\left|\xi_{x i}\right\rangle=[0,1$, $0,-1,0,1,-1,-1,1]^{\top},\left|\xi_{y i}\right\rangle=[0,0,1,0,-1,1,1$, $-1,-1]^{\top}$. Let us collect populations as $\left|f_{i}\right\rangle=$ $\left[f_{0}, f_{1}, f_{2}, f_{3}, f_{4}, f_{5}, f_{6}, f_{7}, f_{8}\right]^{\top}$ (the same will apply to all the equilibrium and post-collision quantities). Following [45], the equilibrium distribution can be expanded as

$$
\begin{aligned}
f_{i}^{(0)}= & w_{i} \rho\left[1+\frac{\boldsymbol{\xi}_{i} \cdot \boldsymbol{u}}{c_{s}^{2}}+\frac{1}{2 c_{s}^{4}} \mathcal{H}_{i}^{(2)}: \boldsymbol{u} \boldsymbol{u}\right. \\
& +\frac{1}{2 c_{s}^{6}}\left(\mathcal{H}_{i x x y}^{(3)} u_{x}^{2} u_{y}+\mathcal{H}_{i x y y}^{(3)} u_{x} u_{y}^{2}\right) \\
& \left.+\frac{1}{4 c_{s}^{8}} \mathcal{H}_{i x x y y}^{(4)} u_{x}^{2} u_{y}^{2}\right],
\end{aligned}
$$

with $w_{0}=4 / 9, w_{1 \ldots 4}=1 / 9$, and $w_{5 \ldots 8}=1 / 36$. As outlined for the D3Q27 space, now we derive the equilibrium CMs for progressively richer expressions of equilibrium $f_{i}^{(0)}$. Let us adopt the basis $\overline{\mathcal{T}}=\left[\bar{T}_{0}, \ldots, \bar{T}_{i}, \ldots, \bar{T}_{8}\right]$ which components are

$$
\begin{aligned}
\left|\bar{T}_{0}\right\rangle & =|1, \ldots, 1\rangle, \quad\left|\bar{T}_{1}\right\rangle=\left|\bar{\xi}_{x i}\right\rangle, \quad\left|\bar{T}_{2}\right\rangle=\left|\bar{\xi}_{y i}\right\rangle, \\
\left|\bar{T}_{3}\right\rangle & =\left|\bar{\xi}_{x i}^{2}+\bar{\xi}_{y i}^{2}\right\rangle, \quad\left|\bar{T}_{4}\right\rangle=\left|\bar{\xi}_{x i}^{2}-\bar{\xi}_{y i}^{2}\right\rangle, \\
\left|\bar{T}_{5}\right\rangle & =\left|\bar{\xi}_{x i} \bar{\xi}_{y i}\right\rangle, \quad\left|\bar{T}_{6}\right\rangle=\left|\bar{\xi}_{x i}^{2} \bar{\xi}_{y i}\right\rangle, \\
\left|\bar{T}_{7}\right\rangle & =\left|\bar{\xi}_{x i} \bar{\xi}_{y i}^{2}\right\rangle, \quad\left|\bar{T}_{8}\right\rangle=\left|\bar{\xi}_{x i}^{2} \bar{\xi}_{y i}^{2}\right\rangle .
\end{aligned}
$$

Let us begin by assuming polynomials up to $\mathcal{H}_{i}^{(2)}$. Then, we obtain

$$
\begin{aligned}
& k_{0}^{(0)}=\rho, \quad k_{3}^{(0)}=2 \rho c_{s}^{2} \quad k_{6}^{(0)}=-\rho u_{x}^{2} u_{y}, \\
& k_{7}^{(0)}=-\rho u_{x} u_{y}^{2}, \quad k_{8}^{(0)}=\rho\left(c_{s}^{4}+u_{x}^{2} u_{y}^{2}\right),
\end{aligned}
$$

with $k_{1,2,4,5}^{(0)}=0$. By considering higher-order polynomials, nonzero equilibrium $\mathrm{CMs}$ become

$$
k_{0}^{(0)}=\rho, \quad k_{3}^{(0)}=2 \rho c_{s}^{2}, \quad k_{8}^{(0)}=\rho\left(c_{s}^{4}-u_{x}^{2} u_{y}^{2}\right),
$$

for $N=3$ and

$$
k_{0}^{(0)}=\rho, \quad k_{3}^{(0)}=2 \rho c_{s}^{2}, \quad k_{8}^{(0)}=\rho c_{s}^{4},
$$

for $N=4$. In the Supplemental Material, a script [48] allows the reader to perform the symbolic manipulations to obtain all the involved quantities. allows the reader to perform the symbolic manipulations to obtain equilibrium CMs in the D2Q9 space. Again, the consistency with the BGK and MRT LBMs is found.
[1] R. Benzi, S. Succi, and M. Vergassola, Phys. Rep. 222, 145 (1992).

[2] S. Succi, The Lattice Boltzmann Equation for Fluid Dynamics and Beyond (Clarendon Press, Oxford, 2001).

[3] S. Succi, Europhys. Lett. 109, 50001 (2015).
[4] S. Succi, Philos. Trans. R. Soc. London A 374, 20160151 (2016).

[5] T. Krüger, H. Kusumaatmaja, A. Kuzmin, O. Shardt, G. Silva, and E. M. Viggen, The Lattice Boltzmann Method: Principles and Practice (Springer, Berlin, Heidelberg, New York, 2016). 
[6] S. Succi, The Lattice Boltzmann Equation: For Complex States of Flowing Matter (Oxford University Press, Oxford, 2018).

[7] P. Bhatnagar, E. Gross, and M. Krook, Phys. Rev. 94, 511 (1954).

[8] J. Latt and B. Chopard, Math. Comput. Simulat. 72, 165 (2006).

[9] D. d'Humières, Philos. Trans. R. Soc. London A 360, 437 (2002).

[10] S. Marié, D. Ricot, and P. Sagaut, J. Comput. Phys. 228, 1056 (2009).

[11] X. B. Nie, X. Shan, and H. Chen, Europhys. Lett. 81, 34005 (2008).

[12] M. Geier, A. Greiner, and J. G. Korvink, Phys. Rev. E 73, 066705 (2006).

[13] M. Geier, A. Greiner, and J. Korvink, Int. J. Mod. Phys. C 18, 455 (2007).

[14] M. Geier, Int. J. Numer. Meth. Fl. 56, 1249 (2008).

[15] P. Asinari, Phys. Rev. E 78, 016701 (2008).

[16] M. Geier, A. Greiner, and J. Korvink, Eur. Phys. J-Spec. Top. 171, 55 (2009).

[17] K. N. Premnath and S. Banerjee, Phys. Rev. E 80, 036702 (2009).

[18] Y. Ning, K. N. Premnath, and D. V. Patil, Int. J. Numer. Meth. Fl. 82, 59 (2016).

[19] M. Geier, M. Schönherr, A. Pasquali, and M. Krafczyk, Comput. Math. Appl. 70, 507 (2015).

[20] A. De Rosis and E. Lévêque, Comput. Math. Appl. 72, 1616 (2016).

[21] M. Geier, A. Pasquali, and M. Schönherr, J. Comput. Phys. 348, 862 (2017).

[22] M. Geier, A. Pasquali, and M. Schönherr, J. Comput. Phys. 348, 889 (2017).

[23] M. Geier and A. Pasquali, Comput. Fluids 166, 139 (2018).

[24] L. Fei and K. H. Luo, Phys. Rev. E 96, 053307 (2017).

[25] L. Fei, K. H. Luo, and Q. Li, Phys. Rev. E 97, 053309 (2018).

[26] N. Shah, P. Dhar, S. K. Chinige, M. Geier, and A. Pattamatta, Numer. Heat Transfer, Part B 72, 211 (2017).

[27] C. S. Kumar, S. Mohankumar, M. Geier, and A. Pattamatta, Int. J. Therm. Sci. 122, 201 (2017).

[28] K. V. Sharma, R. Straka, and F. W. Tavares, Int. J. Therm. Sci. 118, 259 (2017).

[29] L. Fei and K. H. Luo, Int. J. Therm. Sci. 132, 368 (2018).

[30] L. Fei and K. H. Luo, Comput. Fluids 165, 89 (2018).
[31] H. Safari, M. Krafczyk, and M. Geier, Comput. Fluids (2018).

[32] K. Premnath and S. Banerjee, J. Stat. Phys. 143, 747 (2011).

[33] A. De Rosis, Europhys. Lett. 116, 44003 (2016).

[34] A. De Rosis, Phys. Rev. E 95, 013310 (2017).

[35] A. De Rosis, Phys. Rev. E 95, 023311 (2017).

[36] A. De Rosis, Europhys. Lett. 117, 34003 (2017).

[37] A. De Rosis, Phys. Rev. E 96, 063308 (2017).

[38] A. De Rosis, Comput. Method. Appl. M. 319, 379 (2017).

[39] A. De Rosis, E. Lévêque, and R. Chahine, J. Turbul. 19, 446 (2018).

[40] P. J. Dellar, J. Comput. Phys. 179, 95 (2002).

[41] S. Saito, A. De Rosis, A. Festuccia, A. Kaneko, Y. Abe, and K. Koyama, Phys. Rev. E 98, 013305 (2018).

[42] H. Grad, Commun. Pure Appl. Math. 2, 325 (1949).

[43] H. Grad, Commun. Pure Appl. Math. 2, 331 (1949).

[44] X. Shan, X.-F. Yuan, and H. Chen, J. Fluid Mech. 550, 413 (2006).

[45] O. Malaspinas, arXiv:1505.06900.

[46] C. Coreixas, G. Wissocq, G. Puigt, J.-F. Boussuge, and P. Sagaut, Phys. Rev. E 96, 033306 (2017).

[47] C. Coreixas, Ph.D thesis, INP Toulouse, 2018

[48] See Supplemental Material at http://link.aps.org/supplemental/ 10.1103/PhysRevE.99.013301 for performing all the computations to obtain $\left|k_{i}^{(0)}\right\rangle$ [D3Q27_Sixth_order.m]; to perform an extensive von Neumann analysis [D3Q27_StabilityAnalysis.m]; for an animation of the vorticity field of the three-dimensional Taylor-Green vortex [3D_TG_Vortex.avi]; and for performing all the computations to obtain $\left|k_{i}^{(0)}\right\rangle$ in the D2Q9 space [D2Q9_ Fourth_order.m].

[49] D. D'Humieres, Prog. Astronaut. Aeronaut. 159, 450 (1992).

[50] J. D. Sterling and S. Chen, J. Comput. Phys. 123, 196 (1996).

[51] P. Lallemand and L.-S. Luo, Phys. Rev. E 61, 6546 (2000).

[52] P. C. Philippi, L. A. Hegele, Jr., L. O. E. Dos Santos, and R. Surmas, Phys. Rev. E 73, 056702 (2006).

[53] D. N. Siebert, L. A. Hegele, Jr., and P. C. Philippi, Phys. Rev. E 77, 026707 (2008).

[54] M. L. Minion and D. L. Brown, J. Comput. Phys. 138, 734 (1997).

[55] P. J. Dellar, J. Comput. Phys. 259, 270 (2014).

[56] K. K. Mattila, P. C. Philippi, and L. A. Hegele, Jr., Phys. Fluids 29, 046103 (2017).

[57] G. Aubard, P. Volpiani, X. Gloerfelt, and J. Robinet, Flow Turbul. Combust. 91, 497 (2013).

[58] A. Kajzer, J. Pozorski, and K. Szewc, J. Phys.: Conf. Ser. 530, 012019 (2014). 